\title{
SRF Cavity R\&D - Enabling New Frontiers in High Energy Physics and Quantum Science
}

\section{Two Key Figures of Merit}

- Quality factor $\left(Q_{0}\right)$ : improving cavity efficiency, measured by $Q_{0}$ reduces costs for cryogenic plant infrastructure and operation

- Accelerating gradient $\left(E_{a c c}\right)$ : improving gradient makes it possible to reach higher energy

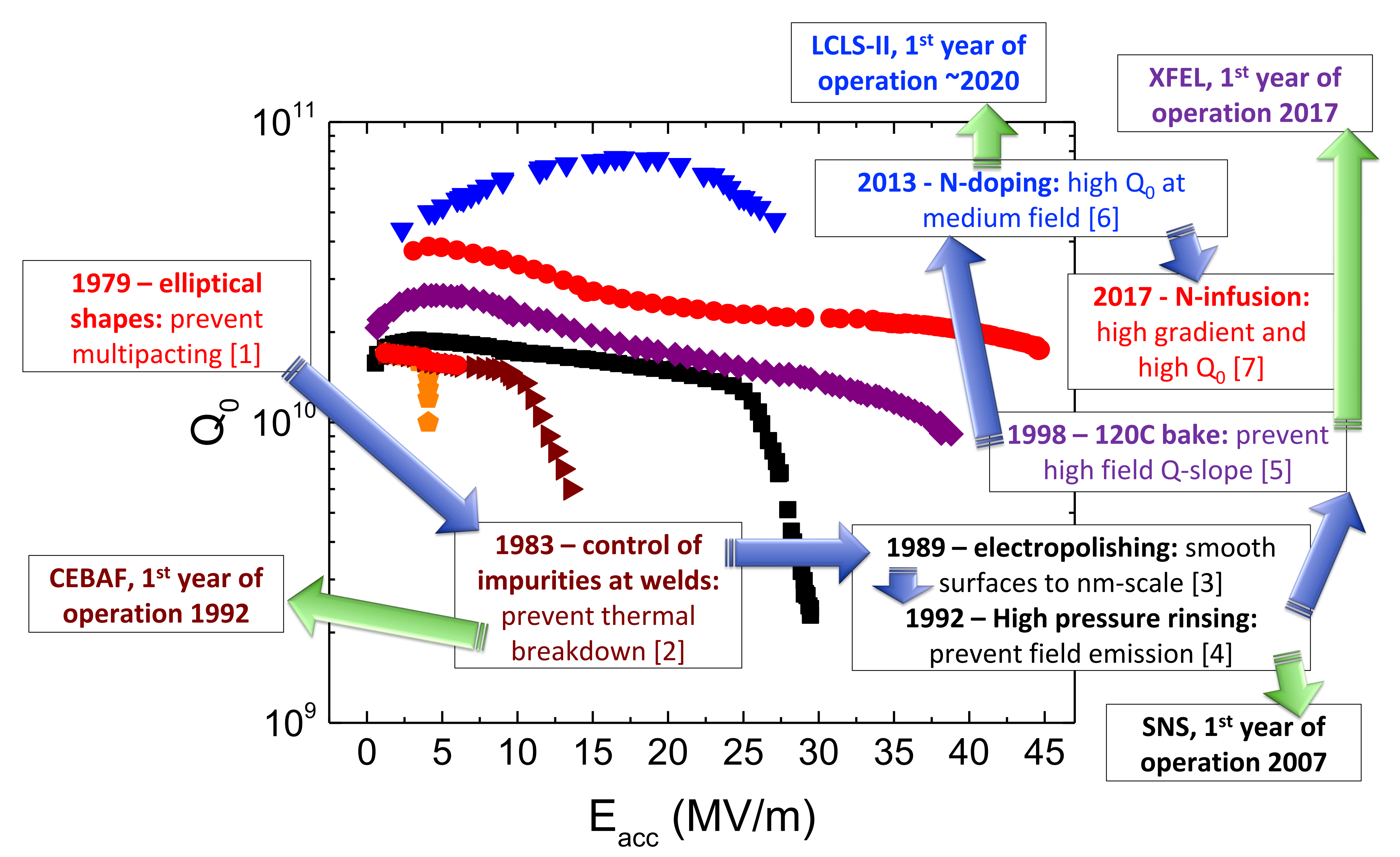

Historical trends of SRF R\&D leading to advances in cavity performance, which then enable new accelerator-based science

- Fermilab SRF R\&D: focus on transformational R\&D for pushing the boundaries of cavity performance

\section{"Megawatts in a Micrometer"}

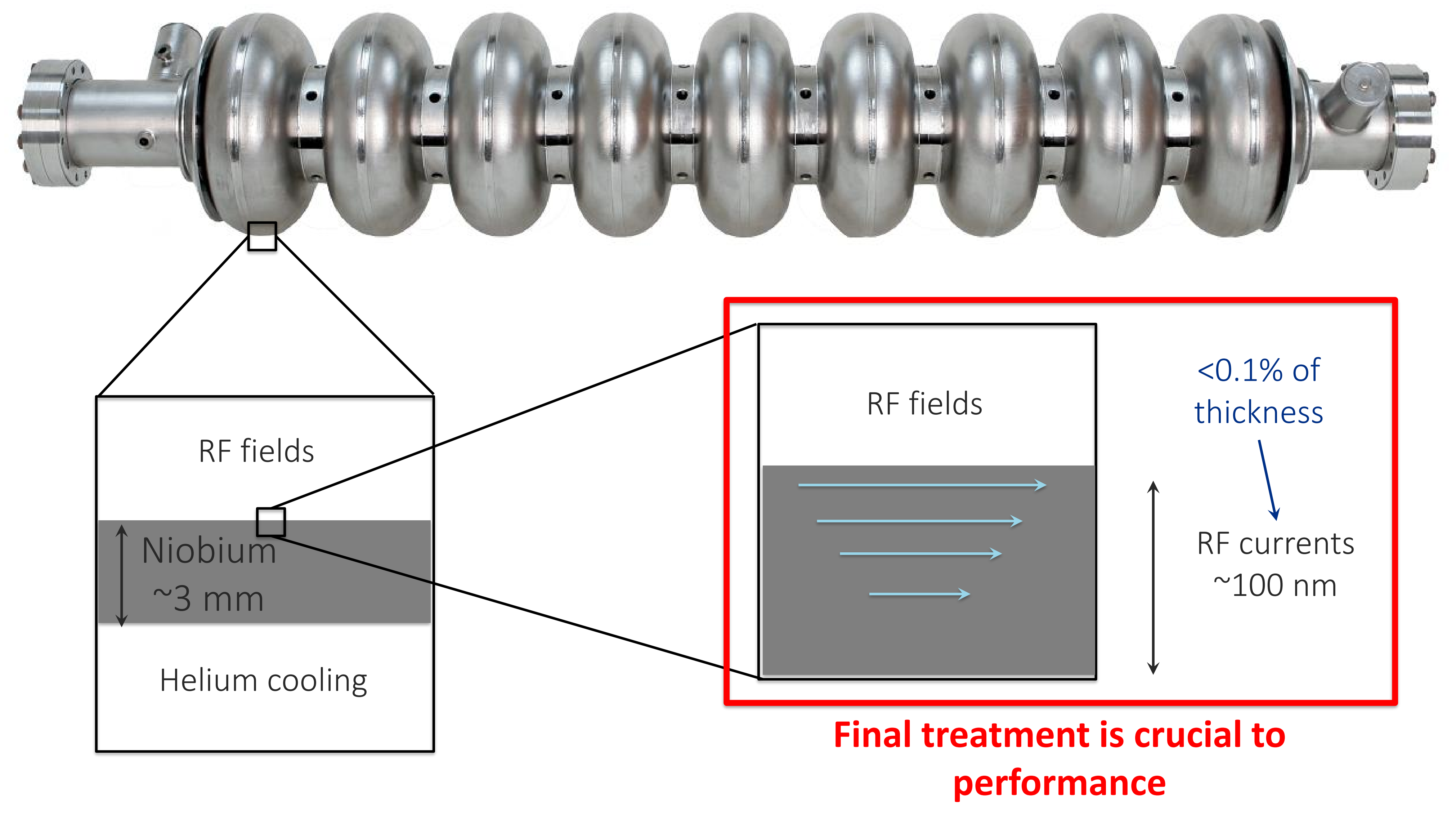

- All RF currents are conducted in $\sim 1$ micrometer layer on inner surface of cavity

- Therefore final treatments have huge impact on $Q_{0}$ and $E_{a c c}$ - performance of an accelerator can be determined by surface processing

This manuscript has been authored by Fermi Research Alliance, LLC under Contract NO. DE-ACO2$07 \mathrm{CH} 11359$ with the U.S. Department of Energy, Office of Science, Office of High Energy Physics.

\section{Materials Science to Connect Performance to Superconductivity}

- Fermilab SRF research often emphasizes materials science, trying to make connections between:

- Surface processing steps

- Microstructure (impurities such as $\mathrm{N}, \mathrm{H}$, and $\mathrm{O}$, dislocations, grain boundaries)

- Cavity performance
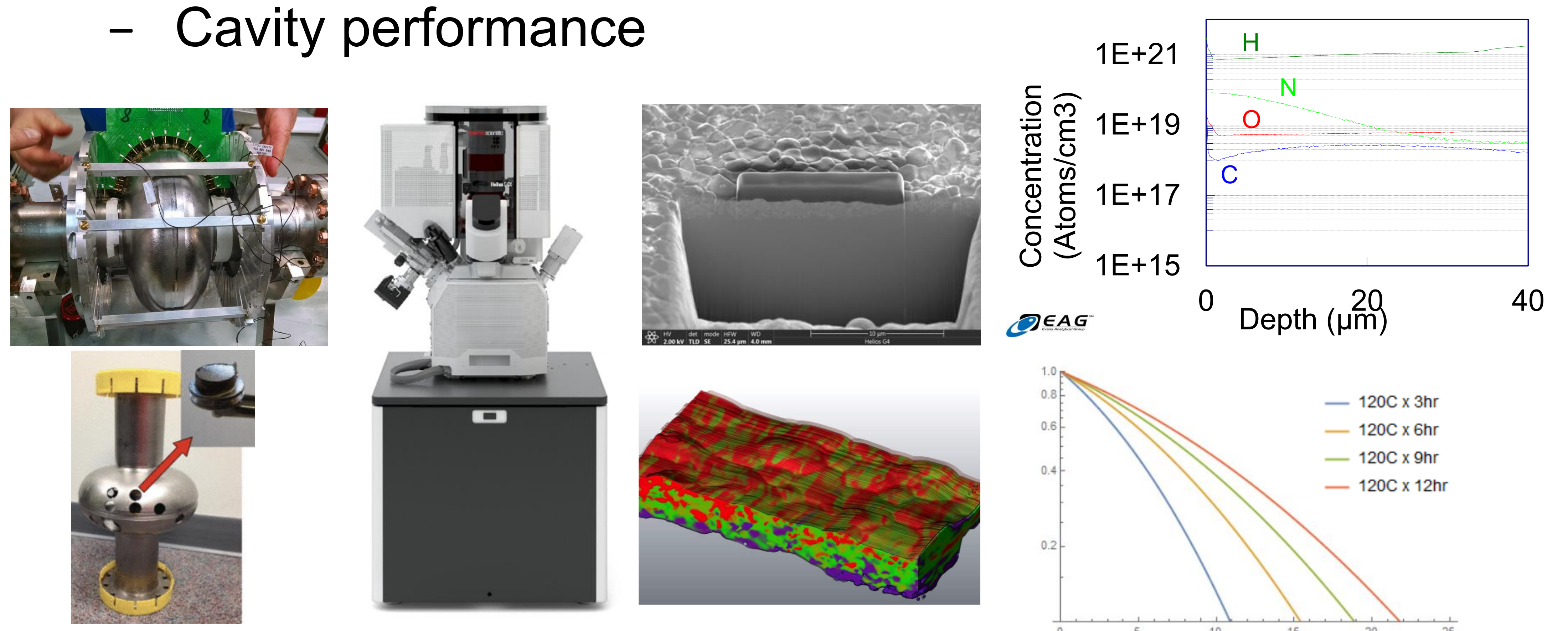

By mapping out dissipation over the surface of a cavity, samples can be prepared with specific knowledge of SRF performance, then analyzed with electron microscopy and other materials science tools

\section{Impacts of Advances in SRF Science}

Advances in SRF research at Fermilab have potential for impact in a wide variety of scientific fields:

- High energy physics (e.g. PIP-II, proposed colliders)

- Basic energy sciences (e.g. LCLS-II, LCLS-II HE)

- Nuclear physics (e.g. proposed MaRIE, EIC)

- Quantum Science (see quantum lab for details)

- Dark matter searches (e.g. ADMX g-2)

- Industrial particle accelerators for wastewater treatment, cancer treatment, isotope production, and more

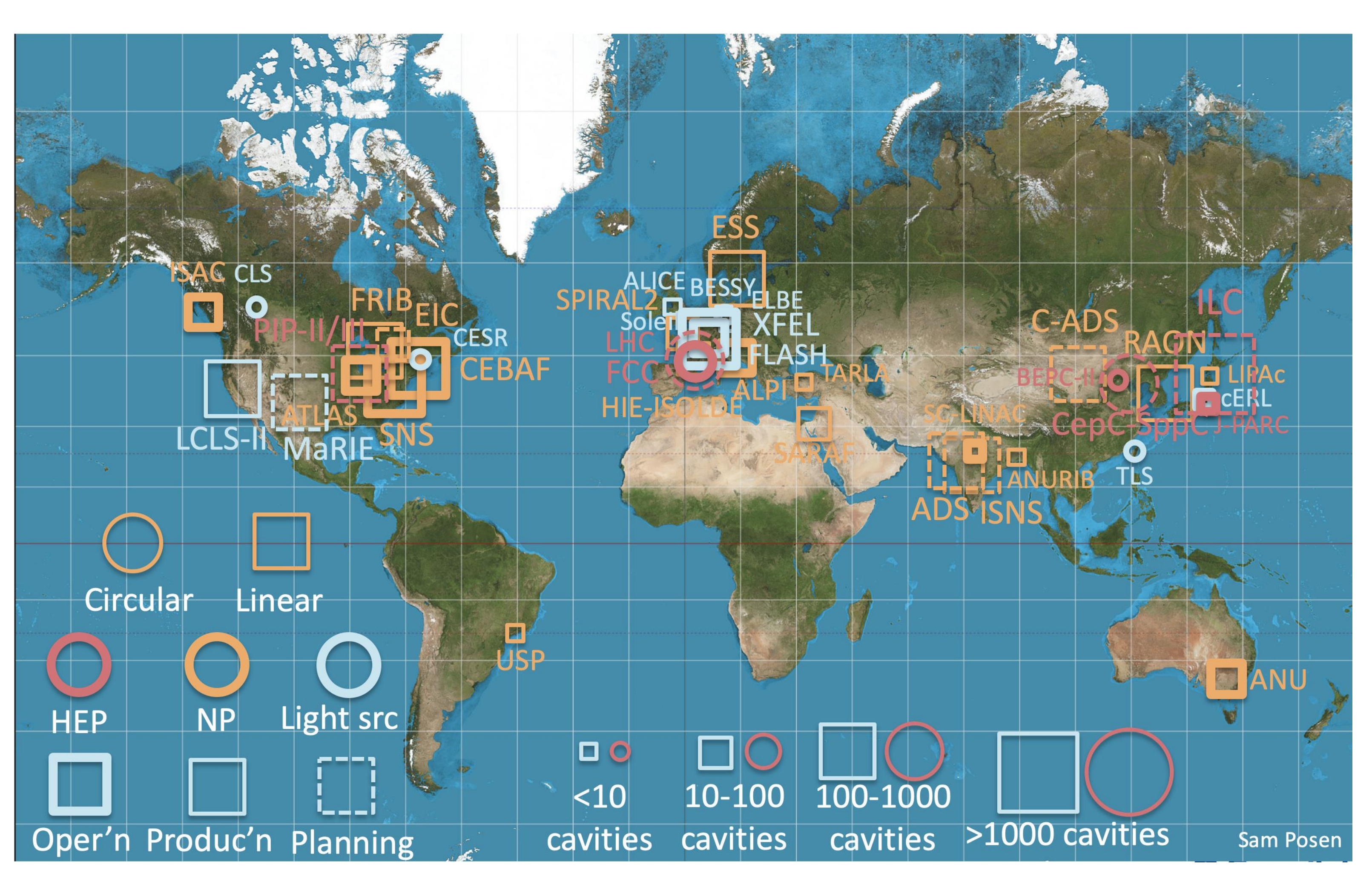

Map of SRF accelerators around the world 